\section{Comércio internacional de serviços e complexo industrial da saúde: implicações para os sistemas nacionais de saúde}

\author{
International trade in health services and the \\ medical industrial complex: implications for \\ national health systems
}

\author{
1 Escola Nacional de Saúde \\ Pública Sergio Arouca \\ Fundação Oswaldo Cruz, Rio \\ de Janeiro, Brasil. \\ 2 Secretaria Municipal de \\ Saúde do Rio de Janeiro, Rio \\ de Janeiro, Brasil. \\ 3 Instituto de Pesquisa \\ Clínica Evandro Chagas, \\ Fundação Oswaldo Cruz, Rio \\ de Janeiro, Brasil. \\ Correspondência \\ M. A. B. Santos \\ Escola Nacional de Saúde \\ Pública Sergio Arouca, \\ Fundação Oswaldo Cruz. \\ Av. Brasil 4036, sala 901, \\ Rio de Janeiro, $R J$ \\ 21040-361, Brasil. \\ angelicabsantos@uol.com.br
}

\begin{abstract}
Health services have increasingly proven to be an innovative sector, gaining prominence in the medical industrial complex through expansion to public and international markets. International trade can foster economic development and redirect the resources and infrastructure available for healthcare in different countries in favorable or unfavorable directions. Wherever private providers play a significant role in government-funded healthcare, GATS commitments may restrict health policy options in subscribing countries. Systematic information on the impacts of electronic health services, medical tourism, health workers' migration, and foreign direct investment is needed on a case-by-case basis to build evidence for informed decision-making, so as to maximize opportunities and minimize risks of GATS commitments.
\end{abstract}

Commerce; Health Care Sector; Medical Economics
Maria Angelica Borges dos Santos 1,2 Sonia Regina Lambert Passos ${ }^{3}$

Recomendações recentes da literatura internacional 1,2,3 reforçam a necessidade de ampliar o conhecimento de profissionais de saúde sobre comércio e globalização, como tentativa de equilibrar os pesos desiguais das agendas da saúde e do comércio em arenas políticas nacionais e internacionais 4,5 .

A discussão sobre as relações entre saúde e comércio não é nova e ganhou projeção recentemente no Brasil com o papel destacado do país na negociação dos anti-retrovirais no âmbito do Trade Related Aspects of Intellectual Property Rights (TRIPS) 1,2. O surgimento do General Agreeement on Trade in Services (GATS) 6, em 1995, integra a agenda de abertura das economias nacionais. Dois aspectos dessa agenda - o comércio internacional e a terceirização - dizem cada vez mais respeito aos serviços de saúde 7 .

As repercussões da agenda liberalizante são freqüentemente analisadas pela literatura em saúde coletiva na perspectiva de suas dimensões políticas ${ }^{8}$. Entretanto, o debate sobre comércio internacional de serviços e outras formas de comercialização de serviços difundidas por essa agenda pode ser complementado por perspectivas analíticas vinculadas a recortes econômicos, como as apresentadas pela discussão do complexo industrial da saúde 9,10.

O complexo industrial da saúde pode ser entendido como a forma particular de estruturação capitalista e expansão mercantil da atenção à saúde 11. Sobre o tema conformou-se uma linha 
de pesquisa que congrega diversos pesquisadores nacionais e um conjunto de teorias explicativas sobre os desenvolvimentos da indústria de saúde 9,10,11,12,13,14,15.

Os estudos clássicos destacam o papel dos fabricantes de insumos e equipamentos como pólo direcionador da estruturação do complexo industrial da saúde 14. Esse segmento, por sua grande capacidade de inovação 9 e acumulação de capital 15 , vem, desde a década de 1950, impondo um padrão de crescente sofisticação tecnológica e necessidade de capitalização à atenção à saúde, que induziu a substituição do padrão liberal de "cuidado e assistência à saúde" por um padrão corporativo de "prestação de serviços de saúde", baseado em empresas 11 .

Entretanto, os serviços de saúde vêm demonstrando, nos últimos anos, um padrão de inovações, discutido na primeira parte deste trabalho, que os credencia a assumir um papel preponderante no complexo industrial da saúde. Segundo as formulações originais, o mecanismo típico de criação de novos mercados para a saúde sustenta-se na legitimidade do prestador de serviços para definir novos parâmetros de consumo ${ }^{16}$, configurando-se o fenômeno da "medicalização" 14 da sociedade. Soma-se agora a esse mecanismo a possibilidade de ampliar mercados pela expansão da presença de serviços mercantis no setor público e pelo comércio internacional dos serviços de saúde.

Com base em uma revisão tradicional de literatura e considerações sobre o padrão de inovações do complexo industrial da saúde, a ampliação de mercados de serviços de saúde e o comércio internacional de serviços de saúde, discutem-se os impactos potenciais da consolidação desse comércio sobre os sistemas de saúde dos países, abordando uma dimensão ainda pouco discutida em âmbito nacional sobre as contradições da agenda para saúde e desenvolvimento econômico 17 .

\section{A dinâmica de inovação do complexo industrial da saúde}

O complexo industrial da saúde é definido de forma variável pelos autores brasileiros que o estudam. Compreende, como núcleo comum reconhecido por todos os autores, atividades econômicas ligadas a setores secundários (fabricação de medicamentos, biofármacos, reagentes diagnósticos e insumos e equipamentos médico-hospitalares e odontológicos) e terciários (prestação de serviços de saúde) ${ }^{14}$. Pode, ainda, incluir a formação de recursos humanos para a saúde 9 , incorporar um complexo médico-financeiro e o
Estado 15, e mais recentemente, como proposta, abranger também um complexo médico-editorial difusor do conhecimento, todos eles com influência sobre o padrão de produção e consumo de atenção à saúde.

A dinâmica de inovação constitui o fator central para a análise das transformações capitalistas e, conseqüentemente, do complexo industrial da saúde 9. Inovar é criar valor pela exploração de alguma forma de mudança baseada em um novo conceito - seja tecnológico, de preços, na regulação ou na geopolítica - gerando novas demandas ou formas de explorar mercados existentes 18 . Assim, a estrutura do complexo industrial da saúde e dos próprios sistemas de saúde é transformada pelas forças de inovação endógenas ao capitalismo, segundo um processo de "destruição criativa ou transformadora", proposto por Schumpeter 19.

A análise do padrão de inovação apresentado pelos dois principais segmentos do complexo industrial da saúde (fabricantes de insumos e prestadores de serviços) nos últimos cinqüenta anos e a relação que estas inovações estabelecem entre esses segmentos permitem-nos acompanhar a dinâmica de transformação do complexo industrial da saúde e dos próprios sistemas de saúde.

Schumpeter 19 estabelece cinco mecanismos para a geração de inovações:

i) Introdução de um novo produto, com o qual os consumidores não estejam familiarizados;

ii) Introdução de um novo método de produção, baseado em uma descoberta cientificamente nova e que pode consistir em uma nova maneira de manejar comercialmente uma mercadoria;

iii) Conquista de uma nova fonte de matérias primas;

iv) Abertura de novos mercados;

v) Estabelecimento de uma forma nova de organização, como a criação de uma posição de monopólio ou a fragmentação de uma posição existente de monopólio.

Em função dos diferenciais de produtividade estabelecidos por Baumol 20 para os setores fabricantes de insumos e prestadores de serviços, pode-se inferir que os primeiros três mecanismos propostos por Schumpeter 19 - centrados no produto - são responsáveis pelas inovações com maior impacto no complexo industrial da saúde do segmento produtor de insumos, ao passo que os dois últimos - centrados no mercado - constituem a contribuição mais importante do segmento prestador de serviços.

Há algumas décadas atuando como pólo impulsionador do complexo industrial da saúde, os produtores de insumos médico-hospitalares incorporaram ciclos sucessivos de inovações ba- 
seadas em todos os mecanismos propostos por Schumpeter 19, a começar pelo desenvolvimento de vacinas, medicamentos e instrumentos diagnósticos.

Com os novos produtos, essas indústrias beneficiaram-se, desde a década de 1950, de uma das mais altas taxas de retorno da economia $15 \mathrm{e}$ da expansão de mercados consumidores propiciada pela consolidação dos welfare states e pelo avanço dos seguros privados. Consolidaram-se também como empreendimentos oligopólicos transnacionais, com tendência incessante a fusões e aquisições 21 .

Schumpeter 22 destaca a importância dos monopólios como motor da inovação e da inovação como remodeladora dos monopólios. Assim, na prática, para se obter lucros é necessário algum tipo de restrição ou monopolização do mercado. Nessa lógica, o mercado seria uma arena em que produtores e vendedores procuram constantemente acabar com os monopólios uns dos outros e empresas altamente capitalizadas lutam para estabelecer mercados monopolistas com alto potencial de inovação 23 .

Uma das expressões contemporâneas da consolidação dos monopólios nos segmentos industriais pode ser interpretada como um reconhecimento da ascensão dos serviços de saúde no complexo industrial da saúde - a integração vertical entre produtores de insumos e prestadores de serviços, capitaneada por produtores de insumos para garantir os mercados para seus produtos. A Fresenius Medical Care, empresa alemã fornecedora de insumos e equipamentos para diálise, transfusão e nutrição enteral, é hoje a líder mundial em serviços de hemodiálise, inclusive no Brasil. No final de 2005, a Fresenius ProServe tornou-se a líder do mercado hospitalar alemão, ao adquirir hospitais do grupo Helius no processo de privatização dos hospitais públicos daquele país 24 .

As estratégias de integração vertical vêm sendo estimuladas pela percepção de uma crise de inovação e da redução real ou projetada dos retornos sobre investimentos do segmento produtor de insumos. O crescimento da avaliação tecnológica para racionalizar custos pode diminuir os mercados consumidores de equipamentos e insumos. Além disso, começam a surgir inseguranças sobre a capacidade da indústria farmacêutica de atrair recursos para pesquisa e desenvolvimento (P\&D) nos patamares atuais, motivadas pela produtividade decrescente da $P \& D$ neste segmento, pela redução da duração das patentes e pela pressão da opinião pública e dos países em desenvolvimento 25 .

A última linha de inovações da indústria farmacêutica-que compreende métodos de produ- ção baseados na genômica e na biotecnologia apresenta resultados ainda decepcionantes. Não foram observados nem incrementos importantes na produtividade nem a geração de novos tipos de fármacos com impactos significativos sobre a saúde. As vendas de biofármacos representam pouco mais de $10 \%$ das vendas de fármacos mundiais, com três quartos delas concentradas em 15 classes terapêuticas de produtos 26 .

A produtividade decrescente do $\mathrm{P} \& \mathrm{D}$ indica uma crise de inovação no setor fabricante de insumos mais importante do complexo industrial da saúde. Segundo um relatório da Consultoria Bernstein citado pela revista The Economist 27, 24 novas drogas foram desenvolvidas com um gasto em P\&D de 27 bilhões de dólares em 1998. Em 2006, a indústria gastou 64 bilhões de dólares para apenas 13 novas drogas aprovadas.

Nesse contexto, passa a ser fundamental acompanhar a dinâmica do setor prestador de serviços para entendermos as transformações contemporâneas do complexo industrial da saúde 10 .

\section{O complexo industrial da saúde e os serviços de saúde}

A prestação de serviços de saúde vem se caracterizando por inovações baseadas em quase todos os mecanismos descritos por Schumpeter 19, com destaque para aquelas que expandem mercados.

Os produtos inovadores - primeiro mecanismo de inovação de Schumpeter - atendem demandas criadas pela redefinição de saúde como bem-estar, ao invés de ausência de doença, e pelo aumento da expectativa de vida. Assim, o princípio estabelecido como fundamento da economia da saúde 16 (p. 948) - o de que a "demanda por serviços médicos não é uniforme e nem previsivel (exceto pela prevenção), pois os serviços médicos só trazem satisfação nos casos de afastamento da situação normal representada pela doença" - já não se sustenta integralmente.

Além do crescimento de produtos ligados à prevenção - dos screenings laboratoriais a check-ups, oferecidos de formas cada vez mais inovadoras e sofisticadas 28 - foram desenvolvidas várias linhas de produtos que atendem à demanda por bem-estar. Essas incluem produtos que melhoram a imagem corporal - cirurgias plásticas, odontologia estética, cirurgia bariátrica - ou vinculadas à medicina reprodutiva e de gênero - como técnicas de fertilização in vitro e cirurgias de mudança de sexo ${ }^{29}$. Além disso, variantes regionais, como a medicina chinesa e a medicina tradicional, surgem como alternativas para o cuidado à saúde. 
Esses novos serviços assumem uma lógica de mercado regular, ligados mais ao desejo dos consumidores do que às necessidades tradicionais de saúde impulsionadas pela doença, descritas por Arrow 16. Com isso, criam-se demandas previsivelmente correlacionadas com níveis de renda da população.

O segundo mecanismo de inovação proposto por Schumpeter 19 são novos métodos de produção, representado, às vezes, por maneiras criativas de conduzir a comercialização. Os exemplos incluem lojas para ofertar check-ups rápidos em shopping centers ${ }^{30}$, a telemedicina e a telecirurgia - que viabilizam a emissão de laudos de exames e a realização de consultas e procedimentos cirúrgicos à distância - além de propostas inovadoras de segmentação de produtos - como hospitais especializados em serviços de saúde para idosos e doenças crônicas, segmentos tradicionalmente desprezados pelo mercado.

Os dois outros tipos de inovação - abertura de novos mercados e mudanças em situações de monopólio - seriam os de maior relevância para o dinamismo contemporâneo do segmento prestador de serviços do complexo industrial da saúde.

O mecanismo típico de criação de novos mercados na saúde sustenta-se na legitimidade do médico para definir novos parâmetros de consumo 16, constituindo o fenômeno da "medicalização" da sociedade 14 . A redefinição periódica de guidelines para doenças freqüentes, como a hipertensão e o diabete, pode ampliar consideravelmente o mercado de pessoas elegíveis para tratamento. Além disso, associações profissionais têm legitimidade para criar uma gama de novos diagnósticos, como exemplificado pela proliferação de subcategorias e gradações para os transtornos de ansiedade, tal como "fobia social”, antes considerada como timidez.

Soma-se agora a esse mecanismo clássico a possibilidade de ampliar mercados pela expansão da presença de serviços mercantis no mercado público - uma inovação baseada na fragmentação de monopólios públicos nacionais para prestação de serviços de saúde - e pela internacionalização dos serviços de saúde, com a criação de novas posições de monopólios privados internacionais.

A fragmentação das situações de monopólio público é capitaneada pelo estímulo a reformas setoriais, com crescente incorporação de prestadores mercantis e terceirização de atividades-fim nos serviços públicos de saúde ${ }^{31}$. Esses prestadores passam a executar funções tradicionalmente executadas pelo setor público, como o tratamento da tuberculose e a administração do Directly Observed Treatment Short-Course (DOTS) 32.
As situações de monopólio privado são fortalecidas pela crescente liberalização dos fluxos de capitais para investimento e pela concentração corporativa. Em 2007, o grupo empresarial americano United Health Group Incorporated atuava em 40 países, contava com 560 mil médicos e 4.800 hospitais. Movimentava quase 100 bilhões de dólares (pouco menos do que o gasto total em saúde brasileiro), com lucros líquidos de 4,7 bilhões de dólares.

No Brasil, um exemplo recente de inovação em serviços de saúde com movimentos simultâneos de criação de monopólios privados e fragmentação de monopólios públicos são os laboratórios de análises clínicas. Até 1998, esses laboratórios compunham um mercado fragmentado com capital quase $100 \%$ nacional e empresas com perfil de gestão familiar. Nos últimos 12, passaram por um processo acelerado de fusões e aquisições, com uma integração horizontal e vertical comandada pelos fornecedores de kits e equipamentos. Os laboratórios menores foram adquiridos pelos grandes e ocorreram fusões dos laboratórios maiores, com o de fundos de capital de risco internacionais 10,33. A partir de 2003, o setor de análises clínicas consolidou-se como um dos primeiros alvos de grande escala da terceirização de atividades - fim no Sistema Único de Saúde (SUS), com experiências já em curso no Rio de Janeiro, Distrito Federal e São Paulo.

As terceirizações de serviços públicos de saúde e o crescimento do comércio internacional de serviços compõem movimentos sinérgicos da dinâmica mais recente de inovação do complexo industrial da saúde.

\section{Acordos de comércio internacional - da regulamentação do comércio de bens à internacionalização dos serviços}

No Manifesto Comunista, Marx \& Engels 34 já analisavam o comércio internacional e a tendência à internacionalização do capital, originada da necessidade de constante expansão dos mercados e do caráter cosmopolita da produção e do consumo. Com o desenvolvimento dos meios de transporte e comunicações, o conceito de competitividade passou a ser definido em âmbito internacional, mais do que local.

O sucesso da internacionalização corresponde à capacidade de controlar e participar de mercados internacionais 35 . Com isso, é possível reduzir custos - ao aumentar a escala de produção, permitir acesso a mão-de-obra e materiais mais baratos e propiciar a instalação de filiais em outros países com possibilidade de pagar menos impostos - e, simultaneamente, ampliar o públi- 
co consumidor, criando, assim, maiores possibilidades de lucros.

Economistas e negociadores na área de comércio reconhecem há muito que as barreiras ao livre comércio não se restringem a tarifas e cotas, mas estão representadas também pelos arcabouços regulatórios econômicos e setoriais nacionais 36 . Desde 1948, com a implantação do General Agreement on Tariffs and Trade (GATT) a partir de um acordo original entre 23 países, existe um movimento no sentido da liberalização e implantação de um ordenamento supranacional do comércio mundial.

Um ordenamento supranacional amplia o acesso a mercados, pois, além de estabelecer padrões mínimos de qualidade obrigatórios que tornam o produto consumível em âmbito global, tende a sobrepor-se a restrições regulatórias nacionais. Na rodada de negociações do Uruguai, de 1994, o GATT foi transformado na Organização Mundial do Comércio (OMC), que conta com 153 países-membros 37 , e cujo arcabouço normativo hoje regula $90 \%$ do comércio mundial 38 .

O crescimento da importância do setor de serviços no comércio internacional acompanha o crescimento de sua participação nas economias nacionais - nos Estados Unidos, em torno de 80\% da economia correspondem a serviços e, no Brasil, mais de $60 \%$ - e avanços nas tecnologias de comunicações e transportes. Ao final da década de 1980, antes do GATS, acordos regionais, como Closer Economic Relations Trade Agreement (CER), o North American Free Trade Agreement (NAFTA) e arranjos no âmbito da União Européia já ensaiavam a liberalização das transações internacionais para serviços 39 .

Com a instituição do GATS, em 1995, cria-se uma regulamentação multilateral legal para o comércio internacional de quase todos os tipos de serviço. $\mathrm{O}$ valor do comércio internacional de serviços cresceu em torno de $6 \%$ ao ano nas décadas de 1980 e 1990, e sua participação no comércio mundial variou de $15,5 \%$, em 1980, para 18,9\%, em 1999 40. Entre 1980 e 2004, o comércio mundial de serviços cresceu mais de $500 \%$ e, hoje, os serviços respondem por $20 \%$ do total de exportações mundiais ou mais de 3 trilhões de dólares 37 .

A atratividade do mercado de serviços de saúde é fornecida por suas dimensões. Os gastos globais com saúde são da ordem de quatro trilhões de dólares, e estima-se que o comércio internacional de serviços de saúde hoje movimente pouco mais de $1 \%$ deste valor. No Brasil, em 2007, os serviços de saúde mercantis faturaram 80 bilhões de reais e o setor público despendeu quase $60 \mathrm{bi}$ lhões de reais para produzir serviços de saúde 41 .

\section{O GATS e os serviços de saúde}

Nos moldes atuais do GATS, os serviços de saúde são divididos em quatro subsetores, que podem ser alvo de graus variáveis de compromisso de liberalização - serviços médicos e dentários; serviços hospitalares; serviços fornecidos por enfermagem, parteiras e outros profissionais; e outros serviços de saúde humana. Os subsetores que atraem mais adesões de compromisso por parte dos países-membros da OMC são os serviços médicos e dentários, seguidos dos hospitalares, ou seja, setores mais intensivos em capital e conhecimento 42 .

O escopo dos serviços reguláveis pelo GATS não se restringe a esses, com impactos direta e imediatamente perceptíveis sobre os serviços de saúde. Inclui também serviços financeiros de seguros de saúde e acidentes; serviços de educação, pesquisa e desenvolvimento em saúde; informática - todos com desdobramentos possíveis para a saúde - e a categoria genérica "serviços de distribuição” que coloca a distribuição de medicamentos e produtos, e equipamentos médicos e terapêuticos sob o escopo potencial do GATS 43 . Numa perspectiva ampliada de saúde pública, o GATS tem também impactos potenciais sobre outras questões ligadas à saúde, como a distribuição de tabaco, qualidade da água e proteção ambiental 2 .

A OMC classifica os serviços comercializáveis de acordo com seus "modos de produção”. Esses quatro modos de produção definem a presença territorial do prestador e do consumidor no momento do fornecimento do serviço, e o tipo de movimentação dos fatores de produção (capital e trabalho) no processo de comercialização 42.

No modo 1, o comércio transfronteira tradicional, consumidor e prestador podem residir em territórios diferentes e são os serviços que cruzam as fronteiras, fornecidos por correio, telefone ou Internet. No caso da saúde, esse modo de produção inclui o envio de amostras biológicas para processamento em outros países e o fornecimento de consultas, laudos e cirurgias à distância - a telemedicina 44 . A demanda global atual estimada por serviços de telemedicina é da ordem de 1,25 trilhão de dólares e pode aumentar com a expansão da infra-estrutura de comunicações 45.

No modo 2, o consumidor desloca-se para o território do prestador, como é o caso de pessoas que procuram atendimento médico ou treinamento profissional em saúde em outros países, seja por falta de oferta em seus países ou em função de melhores condições de oferta fora deles. As novas linhas de serviços de saúde "não essenciais" - cujo consumo é mais previsível do que o 
de produtos tradicionais - são alvos preferenciais desse modo de comercialização. O volume total estimado de importações e exportações nessa modalidade de comercialização em 2003 era de US\$ 11,8 bilhões e, excluída a participação dos Estados Unidos, os países emergentes foram responsáveis por $40 \%$ deste movimento 46 .

No modo 3, o investimento direto estrangeiro (IDE), há um deslocamento de capital, caracterizando presença comercial de uma filial ou subsidiária do prestador de serviços no território do consumidor. Essa é provavelmente a modalidade mais significativa de comércio internacional de serviços na saúde 42,47 . O que constitui IDE pode variar de país a país e incluir porcentual de propriedade em uma empresa $(10 \%$ costumam ser o mínimo, mas o porcentual pode ser definido em mais de metade da propriedade ou, até, em $100 \%)$, administração local ou estrangeira, natureza do investimento ou alguma combinação destes elementos 47 .

Segundo a United Nations Conference on Trade and Development (UNCTAD), para o período 1996 a 2000, enquanto as exportações mundiais aumentaram $4,2 \%$ ao ano, os fluxos de IDE de todas as atividades econômicas cresceram $37 \%$ ao ano e, em 2001, atingiram um valor próximo de 1,3 trilhão de dólares. O processo de internacionalização, no entanto, não foi homogêneo no que diz respeito à participação dos países. Estima-se que, entre 1995 e 2001, o estoque de IDE realizado pelos países desenvolvidos tenha sido oito vezes superior ao realizado no mesmo período pelos países em desenvolvimento 47,48.

A percepção geral é a de que o deslocamento de capital tem viabilizado a formação de grandes oligopólios de prestação de serviços de saúde e bem-estar integrados horizontal e verticalmente. A mídia econômica internacional traz informes recentes sobre movimentos de fusão e compra de grupos de prestadores registrados em vários países, grande parte deles com base em fundos de capital de risco 49 .

O último modo de produção do comércio internacional é o modo 4, caracterizado pelo deslocamento temporário dos profissionais residentes em um país para exercer suas atividades profissionais em outro. Esse é o conhecido brain drain, fenômeno amplamente analisado no relatório da Organização Mundial da Saúde (OMS) de 2006 50. Mais de 30 países pequenos perdem um terço de seus formandos de nível superior para países mais desenvolvidos. A contrapartida é representada por remessas por imigrantes, uma fonte importante de financiamento interno para alguns países.

Existem países já com presença importante em todos os modos de comercialização, como a
Índia, por exemplo. No Brasil, os modos de comércio internacional mais importantes são o 3 (IDE) e, em ascensão, o 2. No nosso país, estimase que o "turismo médico" tenha movimentado cerca de US\$ 600 milhões em 2008, com 40 mil pacientes ao ano 51 , e os mais importantes grupos hospitalares brasileiros privados vêm intensificando um movimento de fusões e investimentos neste nicho de mercado 52 .

\section{Os compromissos no âmbito do GATS}

Em 29 cláusulas, o GATS propõe obrigações gerais, a estrutura de compromissos específicos e instâncias para a resolução de disputas, bem como formula as bases para a progressiva liberalização na área. Com a adesão a acordos internacionais, os países se comprometem a graus de abertura econômica progressivos 53 .

Compromissos firmados no âmbito do GATS têm valor legal e um prazo de validade indefinido. Embora possam ser revistos três anos após o acordo inicial, caso algum país se julgue prejudicado, a quebra de compromissos pode implicar compensação financeira 47 ou render sanções comerciais.

Na primeira etapa de adesão ao GATS, os países comprometem-se a adotar sempre as "medidas” menos restritivas possíveis em relação ao comércio, assegurando-se de que "não excedam o necessário para garantir a qualidade do serviço" 6. "Medidas" incluem, de forma indefinida, padrões técnicos, exigências de licenciamento, regulamentações, leis, subsídios e qualquer outro dispositivo que possa ser interpretado como obstáculo ao livre comércio. Em casos de disputa, a OMC nomeia três membros para determinar se existe desacordo com os termos do GATS. Os membros são especialistas em comércio, mas não necessariamente no setor em pauta e nem na legislação do país em questão ${ }^{2}$.

Como muitos países se opõem a ampliar o alcance do GATS para o setor de serviços, são realizadas rodadas de negociação entre os que subscrevem o acordo para firmarem compromissos de cobertura para cada subsetor de serviços e modo de produção, que podem incluir restrições, desde que claramente enunciadas.

Ao aderir a essa segunda etapa de adesão, o país compromete-se a garantir igualdade de condições ("tratamento nacional") e o "acesso ao mercado" a prestadores estrangeiros de países que subscrevam o acordo no setor. "Acesso ao mercado" inclui o compromisso de não estabelecer limitações na quantidade de prestadores, valor de transações, pessoal empregado, porcentuais de participação de capital estrangeiro e quantidade de operações de serviço 43 . 
A validade dessas regras para serviços de saúde é definida caso a caso no GATS. Na perspectiva do GATS, "serviços" abrangem todos os setores da economia, exceto aqueles "fornecidos no exercício da autoridade governamental”, ou seja, em que inexista base comercial e de competição para oferta do serviço. Onde houver uma combinação de prestadores públicos e privados atuando na saúde pública, como no caso brasileiro, os termos do GATS admitem a interpretação de que a prestação pública recai no âmbito do GATS 44,47,53. Assim, persistem dúvidas sobre como o governo poderia firmar compromissos para a saúde mercantil sem prejudicar a capacidade para exercer "autoridade governamental" na saúde pública 39 .

Uma vez assumido o compromisso com o GATS, todo o setor de saúde, inclusive o público, tende a ser aberto ao comércio e investimento internacional 54 . Assim, não só pode haver participação de grupos privados estrangeiros nas terceirizações de serviços públicos de saúde, mas passam a valer as cláusulas de compromisso de "tratamento nacional" e "acesso a mercados".

Essas regras afetam a governança doméstica tanto quanto o próprio comércio ${ }^{43}$. Instrumentos de planejamento de capacidade usados na saúde pública para regular capacidade instalada e a manutenção de prestadores exclusivamente públicos em qualquer área, podem ser contestados como quebras da cláusula de "acesso a mercados”. Subsídios e isenções de impostos que excluam prestadores estrangeiros constituiriam quebras do compromisso com o "tratamento nacional".

Um agravante adicional seria que somente governos federais são considerados membros do GATS. Governos estaduais e municipais não são reconhecidos como membros, o que torna a representação de outras instâncias federativas nos moldes previstos no SUS inviável ${ }^{2}$.

As implicações do comércio internacional de serviços de saúde podem ser analisadas sob duas perspectivas: a de sua contribuição potencial para o desenvolvimento econômico e a inovação 17, e para rearranjos nos padrões de alocação de recursos e infra-estrutura de saúde entre países e prestadores de serviços públicos e privados; e na perspectiva das implicações da adesão ao GATS para a autonomia dos países de definir suas políticas de saúde.

\section{Implicações do comércio internacional para o desenvolvimento econômico. recursos e infra-estrutura em saúde}

Na perspectiva do desenvolvimento econômico, o argumento a favor do livre comércio de serviços repousa na possibilidade de aumentar as exportações de serviços de saúde e contribuir para fortalecer a balança comercial da saúde, a geração de emprego e a capacidade de inovação pela maior absorção de tecnologia 9,43 .

O comércio internacional pode promover a criação de empregos e renda pelo setor de saúde no país em que se instala. Além disso, as remessas por imigrantes, que incluem profissionais de saúde, e configuram o modo de produção 4 do comércio internacional, são hoje componentes de sustentação importantes de algumas economias periféricas. Em 1999, 3\% das pessoas moravam fora de seus países de nascimento e as remessas monetárias para os países de origem atingiram 300 bilhões de dólares 36 .

A interação entre estruturas internacionalizadas e mais capitalizadas e o mercado de serviços mercantis local pode fomentar a competição, melhorando a qualidade de prestadores e aumentando o acesso a tecnologias. Em outros setores da economia há evidências de efeitos positivos de investimentos estrangeiros no Brasil 55.

Entretanto, como a disponibilidade de recursos tecnológicos, humanos e financeiros para prestação deserviços desaúdeélimitada, asinovações utilizam-se de recursos já disponíveis na sociedade, combinando-os de outras maneiras e retirando-os de locais onde já eram empregados 19.

O desvio de recursos locais para os novos mercados criados pelo comércio internacional poderia, dependendo da direção do comércio, aumentar ou limitar o acesso de parte da população local. Na Índia e Tailândia a alocação de estruturas de produção de serviços de alta complexidade voltadas primordialmente para atendimento do mercado externo tem sido associada à redução de cobertura local 3,56.

Os acordos firmados por países em desenvolvimento para IDE (modo 3) permitem, segundo técnicos da OMC, superar carências de capital humano, promover a eficiência e aumentar a incorporação de novas habilidades e insumos. Entretanto, o IDE consolida a tendência ao surgimento de estruturas crescentemente oligopólicas e, com isto, modifica o poder de barganha dos compradores de serviços públicos ou privados, com impactos sobre a eqüidade 42 .

Uma outra faceta plausível do rearranjo de recursos seria a preservação da oferta pública apenas na medida em que os recursos que mobiliza não pudessem ser empregados de formas 
mais lucrativas nas fatias de mercados emergentes, com um movimento de brain drain ("drenagem de cérebros") interno. Dependendo da estrutura do setor doméstico, poderíamos reforçar a tendência a sistemas de saúde duais, com o aprofundamento das diferenças de qualidade e acesso a serviços específicos entre segmentos populacionais dependentes dos sistemas públicos e privados 57,58 .

Umdesdobramento potencialmenteperverso para a saúde global é o deslocamento de recursos humanos de saúde de regiões mais pobres para países ricos, caracterizado como o modo 4 de comércio internacional. Esse fato é sublinhado pelas recentes preocupações com a disponibilidade e o padrão de migração de recursos humanos para a saúde. A tendência nos países periféricos tem sido um fluxo de saída permanente de pessoal de saúde, com perda do investimento em educação e treinamento destas pessoas 50 .

Uma outra causa bastante em pauta de inquietação é a crescente associação de empreendimentos corporativos de saúde com fundos de capital de risco internacionais 10 . Ainda que possamos estar aliviados pelo fato de não terem sido criados derivativos de mercado, obrigações de saúde colateralizadas e similares, essa hipótese não está de todo afastada, o que transferiria para o setor de saúde considerável vulnerabilidade diante das crises financeiras globalizadas 59 .

O turismo médico (modo 2) e o IDE (modo 3) podem ser particularmente instrumentais para reforçar modelos de atenção em saúde baseados em alto consumo de tecnologias caras. A lei de Say, segundo a qual oferta adicional gera demanda adicional, parece ser sistematicamente válida na saúde 7 .

Uma vez que a configuração dos sistemas nacionais de saúde e as opções políticas dos países variam muito, é bem provável que os efeitos da intensificação do comércio de serviços também variem. São necessárias informações sistematizadas de cada país sobre os impactos de serviços eletrônicos, movimentação internacional de pacientes e profissionais, e investimento direto estrangeiro em serviços de saúde para compor evidências que permitam decisões mais informadas sobre possíveis sinergias entre comércio e saúde, no sentido de potencializar o desenvolvimento e a eqüidade 3 .

Acompanhar a evolução do comércio internacional de serviços, no entanto, não é tarefa fácil 60 . Só recentemente, os governos dos países começam a se preocupar com fontes de informação que permitam mapear a dinâmica internacional desses investimentos e dados pertinentes para outros modos de produção de serviços de saúde contemplados pelo comércio internacional 60. Muitas vezes, sua distribuição e dimensão só podem ser apreendidas com base em informes isolados, de caráter ilustrativo. Essa ausência de informação sistematizada dificulta as análises efetuadas pelos órgãos reguladores com função de equilibrar o poder de mercado de grandes empresas.

As questões fundamentais passariam a ser, então, que tipos de configuração de mercado geram inovações socialmente benéficas e quais são as mais perversas, e como as estruturas de mercado interagem com a formulação de políticas para influenciar a alocação do gasto 61 .

\section{Implicações da adesão ao GATS na autonomia para definir políticas de saúde}

Os riscos impostos pelo GATS à autonomia regulatória e possibilidade de os países definirem autonomamente políticas pertinentes ao financiamento e à oferta de serviços de saúde 43 foram apontados não apenas para países em desenvolvimento, mas para os próprios países desenvolvidos.

Um grande número de autores expressa preocupações especiais quanto a países em desenvolvimento 54,61,62,63,64. A capacidade de regulação desses países costuma ser notadamente insuficiente 61 e, em muitos casos, as empresas transnacionais investidoras podem ser economicamente bem maiores do que os países em que pretendem investir 47 . Em muitos deles, pode ser difícil resistir à pressão de firmas que buscam acesso a mercados de serviço estrangeiros, pois os negociadores de acordos para comercialização de serviços de saúde tendem a ter focos mais difusos do que o das firmas estrangeiras e carecer da informação necessária para garantir desfechos aceitáveis em termos de políticas sociais 39 .

A literatura destaca preocupações com o comprometimento da autonomia dos serviços nacionais de saúde inglês 54 e canadense 65 . Pollock \& Price 54 argumentam que o GATS pode ameaçar a autonomia nacional sobre políticas de saúde, e destacam a necessidade de se criar padrões internacionais e práticas nacionais para proteger os serviços públicos dos efeitos adversos das forças de mercado.

Com a retomada do debate sobre a reforma do sistema de saúde dos Estados Unidos, autores americanos chamam a atenção para o efeito "tiro pela culatra" (ricochet) dos acordos comerciais firmados para facilitar a expansão global da medicina comercial americana. A adesão ao GATS impõe restrições à flexibilidade dos legis- 
ladores americanos e às opções disponíveis para a reforma da saúde, limitando-as a alternativas "orientadas para o mercado", que não permitem excluir a iniciativa privada de nenhum segmento da atenção 43 .

Situação análoga pode estar se configurando no caso brasileiro. Vem crescendo a presença mercantil e os contratos com prestadores de serviço privados - na forma de contratos de gestão para a rede básica e hospitalar e outros modelos de gestão terceirizados, além da delegação da distribuição de medicamentos a redes comerciais de distribuidores no Programa Farmácia Popular. Esses arranjos podem ser interpretados como situações de prestação de serviços públicos de saúde fora "do exercício da autoridade governamental” pelas regras do GATS.

Assim, na hipótese de acordos comerciais para serviços de saúde e serviços de distribuição em geral no escopo do GATS, políticas públicas futuras que envolvessem a prestação de serviços de saúde passariam a ser necessariamente políticas pró-mercado, sob pena de sanções por parte da OMC. No limite, perderíamos a opção de poder excluir prestadores privados nacionais ou internacionais da prestação de serviços pelo poder público, numa flagrante contradição com a legislação do SUS e com as evidências de aumento de custos associados à prestação privada 61 .

A convergência de interesses entre grupos estrangeiros e nacionais de prestadores de serviços de saúde com planos de internacionalização e a política comercial brasileira vigente de incentivo à exportação de serviços podem criar pressões internas no sentido de o Brasil assumir compro- missos no âmbito do GATS para a prestação de serviços de saúde.

Entretanto como, mesmo para economistas 66, à dinâmica econômica sobrepõe-se padrões institucionais e práticas políticas, o desempenho do Brasil com o TRIPS nos autoriza a admitir outros desfechos.

\section{Conclusão}

Dependendo do país em questão, o comércio internacional pode contribuir para o desenvolvimento econômico e para desfechos favoráveis ou desfavoráveis na alocação e localização de recursos e infra-estrutura em saúde. Entretanto, a adesão ao GATS pode restringir as opções políticas dos países para seus sistemas de saúde.

Os principais estudiosos da área recomendam não fechar acordos no âmbito do GATS até a consolidação de mais evidências, caso a caso, sobre os efeitos do comércio internacional para serviços de saúde ${ }^{3}$. Portanto, o tema merece estudos específicos e é recomendável criar indicadores para monitorar o crescimento do comércio internacional.

No Brasil, a reflexão sobre o tema e a participação nas decisões sobre política comercial e externa em áreas com impacto sobre saúde e os serviços de saúde precisam envolver uma gama crescente de atores - profissionais de saúde, Conselhos de Saúde, Agência Nacional de Saúde Suplementar e outras agências reguladoras, corporações profissionais e autoridades da saúde de todos os níveis federativos.

\section{Resumo}

Os serviços de saúde evidenciam um padrão de inovação - na forma de expansão para mercados públicos e do comércio internacional - que os credencia a assumir um papel preponderante no complexo industrial da saúde. O comércio internacional e suas bases regulatórias, definidas no General Agreement on Trade in Services (GATS), têm o potencial de gerar desenvolvimento econômico, mas também de reordenar os recursos disponíveis para a saúde em âmbito nacional e global, em direções favoráveis ou não. Onde houver uma combinação de prestadores públicos e privados atuando na saúde pública, como no caso brasileiro, os termos do GATS admitem a interpretação de que a prestação pública recai no âmbito do GATS, criando-se restrições às opções políticas dos países para a saúde. São necessárias informações sistematizadas e específicas para cada país sobre serviços eletrônicos, movimento de pacientes e profissionais, e investimento direto estrangeiro em serviços de saúde para compor evidências que permitam decisões mais informadas sobre adesão ao GATS.

Comércio; Setor de Assistência à Saúde; Economia Médica 


\section{Colaboradores}

M. A. B. Santos participou da concepção do projeto, redação do artigo, revisão crítica do conteúdo e aprovação final da versão a ser publicada. S. R. L. Passos contribuiu com a concepção do projeto, revisão crítica relevante do conteúdo e aprovação final da versão a ser publicada.

\section{Referências}

1. MacDonald R, Horton R. Trade and health: time for the health sector to get involved. Lancet 2009; 373:273-4.

2. Shaffer ER, Waitzkin H, Breener J, Jasso-Aguillar R. Global trade and public health. Am J Public Health 2005; 95:23-34.

3. Smith RD, Chanda R, Tangcharoensathien Y. Trade in health-related services. Lancet 2009; 373:593601.

4. Fidler DP, Drager N, Lee K. Managing the pursuit of health and wealth: the key challenges. Lancet 2009; 373:325-31.

5. Lee K, Sridhar D, Patel M. Bridging the divide: global governance of trade and health. Lancet 2009; 373:416-22.

6. World Trade Organization. General agreement on trade in services. http://www.wto.org/english/ docs_e/legal_e/26-gats.doc (acessado em 01/ Mar/2010).

7. Waeger P. Trade in health services - an analytical framework. Kiel: Kiel Institute for the World Economy; 2008.

8. Almeida C. Eqüidade e reforma setorial na América Latina: um debate necessário. Cad Saúde Pública 2002; 18 Suppl:23-36.

9. Gadelha CAG. O complexo industrial da saúde e a necessidade de um enfoque dinâmico na economia da saúde. Ciênc Saúde Coletiva 2003; 8:521-35.

10. Andreazzi MFS, Kornis GEM. Padrões de acumulação setorial: finanças e serviços nas transformações contemporâneas de saúde. Ciênc Saúde Coletiva 2008; 13:1409-20.

11. Morone JA. The unruly rise of medical capitalism Hastings Cent Rep 1985; 15:28-31.

12. Relman AS. The new medical-industrial complex. N Engl J Med 1980; 303:963-70

13. Geyman JP. The corporate transformation of medicine and its impact on costs and access to care. J Am Board Fam Pract 2003; 16:443-54.

14. Cordeiro H. As empresas médicas: um estudo das transformações capitalistas da prática médica no Brasil. Rio de Janeiro: Edições Graal; 1984.

15. Vianna CM. Estruturas do sistema de saúde: do complexo industrial ao complexo financeiro. Physis (Rio J.) 2002; 12:375-90.
16. Arrow KJ. Uncertainty and the welfare economics of medical care. Am Econ Rev 1963; 53:941-73.

17. Gadelha CAG. Desenvolvimento e saúde: em busca de uma nova utopia. Saúde Debate 2007; 29:327-38.

18. Lane P. A survey of world trade. The Economist (Suppl) 1998; 3 out.

19. Schumpeter JA. Teoria do desenvolvimento econômico. São Paulo: Editora Abril Cultural; 1982.

20. Baumol WJ. Macroeconomics of unbalanced growth: the anatomy of urban crisis. Am Econ Rev 1969; 57:415-26.

21. Agarwal S, Desai S, Holcomb MM, Oberoi A. Unlocking the value in big pharma. McKinsey Quartely $2001 ;(2): 65-73$

22. Schumpeter JA. Capitalismo, socialismo e democracia. Rio de Janeiro: Jorge Zahar Editor; 1985.

23. Costa $\mathrm{AB}$. O desenvolvimento econômico na visão de Joseph Schumpeter. Cadernos IHU Idéias 2006; (47):1-22.

24. Fresenius Medical Care. Boletim FRA 2006; ano II número 7. http://www.fmc-ag.com.br/profissio nais.html (acessado em 09/Jan/2008).

25. Unit B1-Development of Industrial Policy, Enterprise and Industry Directorate-General, European Commission. European industry: a sectoral overview technical update - 2006. http://ec.europa. eu/enterprise/enterprise_policy/industry/doc/ sec_overview_update06.pdf (acessado em 09/ Out/2008).

26. Hopkins MM, Martin PA, Nightengale P, Kraft A Mahdi S. The myth of the biotech revolution: an assessment of technological, clinical and organizational change. Research Policy 2007; 36:566-89.

27. Beyond the pill. The Economist 2007; 27 out.

28. Costello D. A check-up for retail medicine. Health Aff 2008; 27:1299-303.

29. Henderson JC. Healthcare tourism in Southeast Asia. Tourism Review International 2003; 7:111-22.

30. Laws M, Scott MK. The emergence of retail-based clinics in the United States: early observations. Health Aff 2008; 27:1293-8.

31. Preker S. Managing scarcity through strategic purchasing of health care. In: Preker AS, Langenbrunner JC, editors. Spending wisely: buying health services for the poor. Washington DC: The World Bank; 2005. p. 23-60. 
32. Evans P. Use of contracting in public health. Bull World Health Organ 2006; 84:842.

33. Cavalcanti RLS. Globalização na área de saúde: os processos de aquisição e fusão dos laboratórios de análises clínicas e suas implicações. Rio de Janeiro: Qualitymark; 2005.

34. Marx K, Engels F. Manifesto do partido comunista. São Paulo: Editora Martin Claret; 2004.

35. Tachinardi MHA. Guerra das patentes. Rio de Janeiro: Editora Paz e Terra; 1993.

36. Beedham B. A survey on new geopolitics The Economist (Suppl) 1999; 31 jul.

37. World Trade Organization. International trade statistics 2008. http://www.wto.org/english/res_e/ statis_e/its2008_e/its08_toc_e.htm (acessado em 01/Dez/2008).

38. Bettcher DW, Yach D, Guidon GE. Global trade and health: key linkages and future challenges. Bull World Health Organ 2000; 78:521-34.

39. Hoekman B. Liberalizing trade in services: a survey. Washington DC: World Bank; 2006. (World Bank Policy Research Working Paper, 4030).

40. Woodward D, Drager N, Beaglehole R, Lipson D. Globalization, global public goods and health. In: Drager N, Vieira C, editors. Trade in health services: global, regional and country perspectives. Washington DC: Division of Health and Human Development, Program on Public Policy and Health, Pan American Health Organization; 2002. p. 3-11.

41. Instituto Brasileiro de Geografia e Estatística. Conta-satélite de saúde: Brasil: 2005-2007. Rio de Janeiro: Instituto Brasileiro de Geografia e Estatística; 2009.

42. Adlung R, Carzaniga A. Health services under the General Agreement on Trade Services. Bull World Health Organ 2001; 79:352-64.

43. Arnold PJ, Reeves TC. International trade and health policy: implications of the GATS for US healthcare reform. J Bus Ethics 2006; 63:313-32.

44. Chandra R. Trade in health services. Bull World Health Organ 2002; 80:158-63.

45. Mutchnick IS, Stern D, Moyen CA. Trading services across borders: GATS, markets and caveats. Health Aff 2005; 24:542-51.

46. Lautier M. Export of health services from developing countries: the case of Tunisia. Soc Sci Med 2008; 67:101-10.

47. Smith RD. Foreign direct investment and trade in health services: a review of the literature. Soc Sci Med 2004; 59:2313-23.

48. Corrêa D, Lima GT. O comportamento recente do investimento direto brasileiro no exterior em perspectiva. Revista de Economia Política 2008; 28: 249-68.

49. Marz A. El hospital privado ya es un producto de masas. El País 2008. http://www.elpais.com/artic ulo/sociedad/hospital/privado/producto/masas/ elpepusoc/20080522elpepisoc_1/Tes (acessado em 30/Set/2008).

50. World Health Organization. The World Health Report 2006 - working together for health. http:// www.who.int/whr/2006/en/ (acessado em 23/Dez/ 2008).
51. Diário Comércio, Indústria \& Serviços. Turismo de saúde terá giro de US\$ 600 milhões. http://vpaper. dci.com.br/default.asp?ed=605 (acessado em 13/ Set/2009).

52. Valor Econômico. Sírio-Libanês investe para atender mais estrangeiro. http://www.investi mentos.sp.gov.br/noticias/lenoticia.php?id= $3663 \& \mathrm{c}=6 \&$ lang $=1$ (acessado em 01/Mar/2010).

53. Fidler DP, Correa C, Aginam O. Legal review of the General Agreement on Trade in Services (GATS) from a health policy perspective: globalization, trade and health. Geneva: World Health Organization; 2005.

54. Pollock AM, Price D. Rewriting the regulations: how the World Trade Organisation could accelerate privatisation in health-care systems. Lancet 2000; 356:1995-2000.

55. Chudnovsky D, López A. Inversión extranjera directa y desarrollo: la experiencia del Mercosur. Revista de la CEPAL 2007; (92):7-23.

56. Chinai R, Goswami R. Medical visas mark growth of Indian medical tourism. Bull World Health Organ 2007; 85:164-5.

57. Santos MAB, Gerschman S. As segmentações da oferta de serviços de saúde no Brasil: arranjos institucionais, credores, pagadores e devedores. Ciênc Saúde Coletiva 2004; 9:795-806.

58. Pachanee C, Wibulpolprasert S. Incoherent policies on universal coverage of health insurance and promotion of international trade in health services in Thailand. Health Policy Plan 2006; 21:310-8.

59. Dentzer S. Medical technology \& spending: the next market bubble? Health Aff 2008; 27:1464-5.

60. Holden C. Investigating international health markets: methodological problems and challenges. Social Policy and Society 2005; 4:427-35.

61. Mackintosh M, Koivusalo M. Health systems and commercialisation. In search of good sense. In: Mackintosh M, Koivusalo M, editors. Commercialisation of health care: global and local dynamics and policy responses. Basingstoke: Palgrave; 2005. p. 3-21.

62. Walt G. Globalization and international health. Lancet 1998; 351:434-7.

63. Pollock AM, Price D. The public health implications of world trade negotiations on the general agreement on trade in services and public services. Lancet 2003; 362:1072-5.

64. Blouin C. Trade policy and health: from conflicting interests to policy coherence. Bull World Health Organ 2007; 85:169-73.

65. Sanger M, Sinclair S. Putting health first: Canadian health care reform, trade treaties and foreign policy. Ottawa: Canadian Centre for Policy Alternatives Consortium on Globalization and Health; 2002.

66. Stigliz JE. Globalization and its discontents. New York: Norton Press; 2002.

Recebido em 17/Jan/2009

Versão final reapresentada em 03/Mar/2010

Aprovado em 11/Jun/2010 